\title{
Abstract: nnU-Net: Self-adapting Framework for U-Net-Based Medical Image Segmentation
}

\author{
Fabian Isensee ${ }^{1}$, Jens Petersen ${ }^{1}$, Andre Klein ${ }^{1}$, David Zimmerer ${ }^{1}$, \\ Paul F. Jaeger ${ }^{1}$, Simon Kohl ${ }^{1}$, Jakob Wasserthal ${ }^{1}$, Gregor Koehler ${ }^{1}$, \\ Tobias Norajitra ${ }^{1}$, Sebastian Wirkert ${ }^{1}$, Klaus H. Maier-Hein ${ }^{1}$ \\ ${ }^{1}$ Department of Medical Image Computing, German Cancer Research Center, \\ Heidelberg, Germany \\ f.isensee@dkfz-heidelberg.de
}

The U-Net was presented in 2015. With its straight-forward and successful architecture it quickly evolved to a commonly used benchmark in medical image segmentation. The adaptation of the U-Net to novel problems, however, comprises several degrees of freedom regarding the exact architecture, preprocessing, training and inference. These choices are not independent of each other and substantially impact the overall performance. The present paper [1] introduces the nnU-Net ("no-new-Net"), which refers to a robust and self-adapting framework on the basis of $2 \mathrm{D}$ and $3 \mathrm{D}$ vanilla U-Nets. We argue the strong case for taking away superfluous bells and whistles of many proposed network designs and instead focus on the remaining aspects that determine the performance and generalizability of a method. We evaluate the nnU-Net in the context of the Medical Segmentation Decathlon challenge, which measures segmentation performance in ten disciplines comprising distinct entities, image modalities, image geometries and dataset sizes. Most importantly though, algorithms submitted to this challenge are required to work out of the box for any of these datasets without manual intervention or fine tuning. The challenge is divided in two distinct phases: phase I comprises seven datasets and is mainly intended for model development while phase II comprises three previously unknown datasets indented for model evaluation. nnUNet successfully adapted itself to all of these datasets without user interaction and, with the sole exceptions being class 1 in the BrainTumour and the Spleen datasets, achieved the highest dice scores out of all participating algorithms (phase I leaderboard: https://decathlon.grandchallenge.org/evaluation/results/). In the final evaluation it won the Medical Segmentation Decathlon challenge with a margin.

\section{References}

1. Isensee F, Petersen J, Klein A, et al. nnU-Net: self-adapting framework for U-Netbased medical image segmentation. arXiv:180910486. 2018;. 